\title{
Article \\ Spectrum of Kidney Disorders Associated with T-Cell Immunoclones
}

\author{
Alexis Piedrafita ${ }^{1,2}$, François Vergez ${ }^{3,4}$ (D) Julie Belliere ${ }^{1,2,4}$, Nais Prades ${ }^{3}$, Magali Colombat ${ }^{4,5}$, Antoine Huart ${ }^{1}$, \\ Jean-Baptiste Rieu ${ }^{3}$, Stéphanie Lagarde ${ }^{3}$, Arnaud Del Bello 1,4, Nassim Kamar 1,4 (D), Dominique Chauveau 1,2,4, \\ Camille Laurent ${ }^{4,5}$, Lucie Oberic ${ }^{5}$, Loïc Ysebaert ${ }^{4,6}$, David Ribes ${ }^{1}$ and Stanislas Faguer ${ }^{1,2,4, *}$
}

check for updates

Citation: Piedrafita, A.; Vergez, F.; Belliere, J.; Prades, N.; Colombat, M.; Huart, A.; Rieu, J.-B.; Lagarde, S.; Del Bello, A.; Kamar, N.; et al. Spectrum of Kidney Disorders Associated with T-Cell Immunoclones. J. Clin. Med. 2022, 11, 604. https://doi.org/ $10.3390 /$ jcm11030604

Academic Editor: Pasquale Esposito

Received: 15 December 2021

Accepted: 24 January 2022

Published: 25 January 2022

Publisher's Note: MDPI stays neutral with regard to jurisdictional claims in published maps and institutional affiliations.

Copyright: (C) 2022 by the authors. Licensee MDPI, Basel, Switzerland. This article is an open access article distributed under the terms and conditions of the Creative Commons Attribution (CC BY) license (https:// creativecommons.org/licenses/by/ $4.0 /)$.
1 Centre de Référence des Maladies Rénales Rares, Département de Néphrologie et Transplantation d'Organes, Centre Hospitalier Universitaire de Toulouse, F-31000 Toulouse, France; piedrafita.a@chu-toulouse.fr (A.P.); belliere.j@chu-toulouse.fr (J.B.); huart.a@chu-toulouse.Fr (A.H.); delbello.a@chu-toulouse.fr (A.D.B.); kamar.n@chu-toulouse.fr (N.K.); chauveau.d@chu-toulouse.fr (D.C.); ribes.d@chu-toulouse.fr (D.R.)

2 UMR 1297 (Institut des Maladies Métaboliques et Cardiovasculaires-Team 12), Institut National de la Santé et de la Recherche Médicale, F-31000 Toulouse, France

3 Laboratoire d'Hématologie, Institut Universitaire du Cancer de Toulouse-Oncopôle, Centre Hospitalier Universitaire de Toulouse, F-31000 Toulouse, France; vergez.Francois@iuct-oncopole.fr (F.V.); prades.nais@iuct-oncopole.fr (N.P.); rieu.jean-baptiste@iuct-oncopole.fr (J.-B.R.); lagarde.stephanie@iuct-oncopole.fr (S.L.)

4 Faculté de Médecine Rangueil, Université Paul Sabatier-Toulouse III, F-31000 Toulouse, France; colombat.magali@iuct-oncopole.fr (M.C.); laurent.camille@iuct-oncopole.fr (C.L.); ysebaert.loic@iuct-oncopole.fr (L.Y.)

5 Département d'Anatomopathologie, Institut Universitaire du Cancer de Toulouse, Centre Hospitalier Universitaire de Toulouse, F-31000 Toulouse, France; oberic.lucie@iuct-oncopole.fr

6 Service d'Hématologie, Institut Universitaire du Cancer de Toulouse-Oncopôle, Centre Hospitalier Universitaire de Toulouse, F-31000 Toulouse, France

* Correspondence: stanislas.faguer@inserm.fr; Tel.: +33-561-323-288; Fax: +33-561-322-351

Abstract: Large granular T-cell leukemia is a clonal hematological condition often associated with autoimmune disorders. Whether small-sized T-cell clones that are otherwise asymptomatic can promote immune kidney disorders remains elusive. In this monocentric retrospective cohort in a tertiary referral center in France, we reviewed characteristics of 29 patients with T-cell clone proliferation and autoimmune kidney disorders. Next-generation sequencing of the T-cell receptor of circulating T-cells was performed in a subset of patients. The T-cell clones were detected owing to systematic screening (mean count $0.32 \times 10^{9} / \mathrm{L}$, range 0.13-3.7). Strikingly, a common phenotype of acute interstitial nephropathy was observed in 22 patients (median estimated glomerular filtration rate at presentation of $22 \mathrm{~mL} / \mathrm{min} / 1.73 \mathrm{~m}^{2}$ (range 0-56)). Kidney biopsies showed polymorphic inflammatory cell infiltration (predominantly $\mathrm{CD}^{+} \mathrm{T}$-cells, most of them demonstrating positive phospho-STAT3 staining) and non-necrotic granuloma in six cases. Immune-mediated glomerulopathy only or in combination with acute interstitial nephropathy was identified in eight patients. Next-generation sequencing $(n=13)$ identified a major T-cell clone representing more than $1 \%$ of the T-cell population in all but two patients. None had a mutation of STAT3. Twenty patients (69\%) had two or more extra-kidney autoimmune diseases. Acute interstitial nephropathies were controlled with corticosteroids, cyclosporin A, or tofacitinib. Thus, we showed that small-sized T-cell clones (i.e., without lymphocytosis) undetectable without specific screening are associated with various immune kidney disorders, including a previously unrecognized phenotype characterized by severe inflammatory kidney fibrosis and lymphocytic JAK/STAT activation.

Keywords: immunoclones; large granular lymphocytic leukemia; renal fibrosis; T-cell receptor; STAT3; autoimmune disorders 


\section{Introduction}

The development of new molecular diagnosis tools, including multicolor flow cytometry, polymerase chain reaction of the T-cell receptor (TCR), and next-generation sequencing, has provided new opportunities to refine the phenotypes and molecular mechanisms of immune disorders of unknown origin. This approach helped to relate large granular lymphocytic leukemia (LGL), a condition frequently associated with autoimmune diseases, with a clonal or oligoclonal proliferation of cytotoxic T-cells [1].

LGL is considered a reactive process with extreme clonal skewing [1,2]. Most LGL cells harbored a $\mathrm{CD}^{+} \mathrm{CD}^{+}$phenotype, and more rarely expressed the CD4 marker, or had a natural killer (NK) phenotype [1]. Activation of the JAK/STAT pathway in CD8 ${ }^{+}$ T-cells is observed in most if not all LGL clones and is driven by acquired mutations of the STAT3 or very rarely STAT $5 b$ genes in $5 \%$ to $30 \%$ of patients [3-5]. STAT3 mutations are considered a secondary event during clonal expansion, but LGL can evolve and progress because of persistent antigenic drive, even if STAT3 is not mutated [4].

Cytopenia (autoimmune hemolytic anemia and neutropenia) and rheumatoid arthritis are the most frequent immune disorders associated with LGL, but a wide panel of autoimmune diseases were also observed in patients with LGL, including hematological (red cell aplasia, pernicious anemia, thrombocytopenia), neurological (myasthenia gravis, polyradiculoneuropathy), or rheumatic (Sjögren's syndrome, lupus) disorders [1,2]. To date, only seven cases of renal disease related to LGL have been reported, including renal infiltration by the LGL or heterogeneous glomerulopathies (focal and segmental glomerulosclerosis, vasculitis with anti-glomerular basement membrane antibodies, heavy-chain amyloidosis, glomerulonephritis with endocapillary proliferation) [6-12]. Recently, we reported two cases of inflammatory renal fibrosis associated with a small-sized T-cell clone (i.e., a clonal population not associated with lymphocytosis and thus requiring dedicated immunophenotyping to be identified) [13]. We demonstrated that these immunoclones may promote phenotypic changes of the epithelial renal cells favoring renal fibrosis. In both cases, steroids' responsiveness was dramatic. Bone marrow fibrosis induced by the LGL clone was also previously demonstrated [14], but whether such small-sized LGL proliferation may promote multi-organ inflammatory fibrosis remains elusive. Previous studies using multicolor flow cytometry also suggested that the size of the circulating LGL clone is highly heterogeneous and may vary from 0.1 to $>10 \times 10^{9} / \mathrm{L}$, supporting the use of a new functional definition based on the need to (a) treat the immunologic effects of the immunoclones (T-cell immunoclones, TIC), (b) to treat the neoplastic effects of leukemia (LGL leukemia), or (c) to only monitor the patients with an asymptomatic circulating T-cell clone (asymptomatic patients, T-cell clone of undetermined significance (TCUS)) [15-17].

In this study, we aimed to describe the immune kidney disorders associated with T-cell immunoclones.

\section{Methods}

In this retrospective monocentric study, we included all adult patients with a Tcell immunoclone detectable in the blood or organ and followed at the department of Nephrology, Clinical Immunology, and Organ Transplantation of the University Hospital of Toulouse (France). The inclusion period was January 2010 to September 2020. The study was conducted in compliance with the Good Clinical Practice protocol and the Declaration of Helsinki principles, as well as French law regarding retrospective observational studies. According to the recommendations of the Institutional Review Board of the University Hospital of Toulouse, written informed consent was waived.

\subsection{Patients' Characteristics}

Clinical and biological parameters were collected through a standardized screening of the patients' hospital records. Routine kidney pathology consisted of light microscopy and immunostaining directed against IgG, IgA, IgM, kappa and lambda light chains, CD3, and CD20. The expression of phospho-STAT3 was performed on $3 \mu \mathrm{m}$-thick sections of 
available formalin-fixed paraffin-embedded whole-tissue biopsies of the kidney, liver, or lymph node and tested using the Ventana Benchmark XT immunostainer (Ventana, Tucson, AZ, USA). Samples were then stained with the anti-phospho-STAT3 antibody (dilution 1/100; RM261; Diagomics, Blagnac, France). Co-immunostaining could not be performed due to the small amount of tissue available, but all biopsies were reviewed by a pathologist (C.L.) to assess whether the cellular compartment p-STAT3 was identified (endothelial, epithelial, lymphoid cells).

\subsection{Immunological Analyses}

Indication to screen for immunoclones was lymphocytosis $\left(>5000 / \mathrm{mm}^{3}\right)$, autoimmunity, or inflammatory fibrosis of unknown origin. Blood samples were collected in EDTA tubes and usually tested within $48 \mathrm{~h}$ of collection. The quantification of lymphocyte subpopulations was determined by flow cytometry using DuraClone IM phenotyping Basic Tubes and Flow Fluorospheres (Beckman Coulter, Brea, CA, USA). Briefly, the cells were stained with a panel of antibodies targeting CD3 (clone UCHT1), CD5 (clone L17F12), CD56 (clone NCAM16. 2), CD57 (clone HNK-1), HLA-DR (clone G46-6) (BD Biosciences, Franklin Lakes, NJ), CD16 (clone 3G8), CD8 (clone B9.11) (Beckman Coulter, Brea, CA, USA), and CD4 (clone RPA-T4) (Biolegend, San Jose, CA, USA). Flow cytometry data were acquired on a Navios instrument and analyses were performed with Kaluza software (Beckman Coulter, Brea, CA, USA).

Diagnosis of immunoclones relied on the identification of a circulating lymphocyte population with immune phenotype characteristics of LGLT, LGLNK, or CD4+ T-cell clones, or with a demonstration of organ infiltration by a T-cell clone. T-cell clonality was studied on DNA extracted from circulating cells or frozen tissue by multiplex PCR targeting the TRB and TRG genes, following DNA quality assessment by a control ladder PCR. Biomed-2 primers were used with standard PCR conditions. Amplification products were analyzed by electrophoresis on polyacrylamide gel.

\subsection{STAT3 and TCR Sequencing}

To estimate the size of the immunoclone population and search for STAT3 acquired mutations, next-generation sequencing of the T-cell receptor (TCR) Vd and Jd complementaritydetermining region 3 (CDR3) and the STAT3 gene was performed with PCR amplicon-based libraries. The amplicons were generated by specific multiplexed PCRs, one for STAT3 exon 20 and 21, and two for TCR $\gamma$ and TCR $\beta$ rearrangements (primers based on the Biomed-2 European collaborative study [18]). The obtained PCR amplicons were then subjected to an indexing PCR with the Nextera XT Index kit V2 (Illumina, San Diego, CA, USA). The libraries were sequenced with a MiSeq sequencer (Illumina, San Diego, CA, USA) and Miseq Reagent kit V2 (paired-end sequencing $2 \times 150$ cycles). For STAT3 mutations' analysis, alignment was performed using the BWA aligner and variant calling was performed using FreeBayes and Mutect2 variant callers. For TCR rearrangements' alignment and detection, we used the Vidjil web platform [19].

\subsection{Statistical Analyses}

Data were shown as medians and limits for continuous variables, and as numbers and percentages for discontinuous variables.

\section{Results}

\subsection{Patients}

From January 2010 to November 2020, a chronic LGL population was identified in 55 patients, including 10 solid organ transplant recipients (accounting for $21 \%$ of the total peripheral blood lymphocyte counts $(7 ; 51)$ ) (Figure 1). The circulating clone was identified during the screening of asymptomatic lymphocytosis in 13 patients $(24 \%$, including nine solid organ transplant recipients), leading to the diagnosis of TCUS. The remaining 42 patients had TIC. In them, the T-cell clone was searched for to explore the mechanisms of 
polyautoimmunity or immune-mediated nephropathy. One kidney transplant recipient had recurrent episodes of acute interstitial nephropathy, formerly considered as acute cellular rejection, and myositis. No TCUS patient developed overt T-cell lymphoma, B symptoms, or splenomegaly, and none received anti-lymphoma therapy. Among the 13 TCUS cases, 11 were considered reactive LGL proliferation.

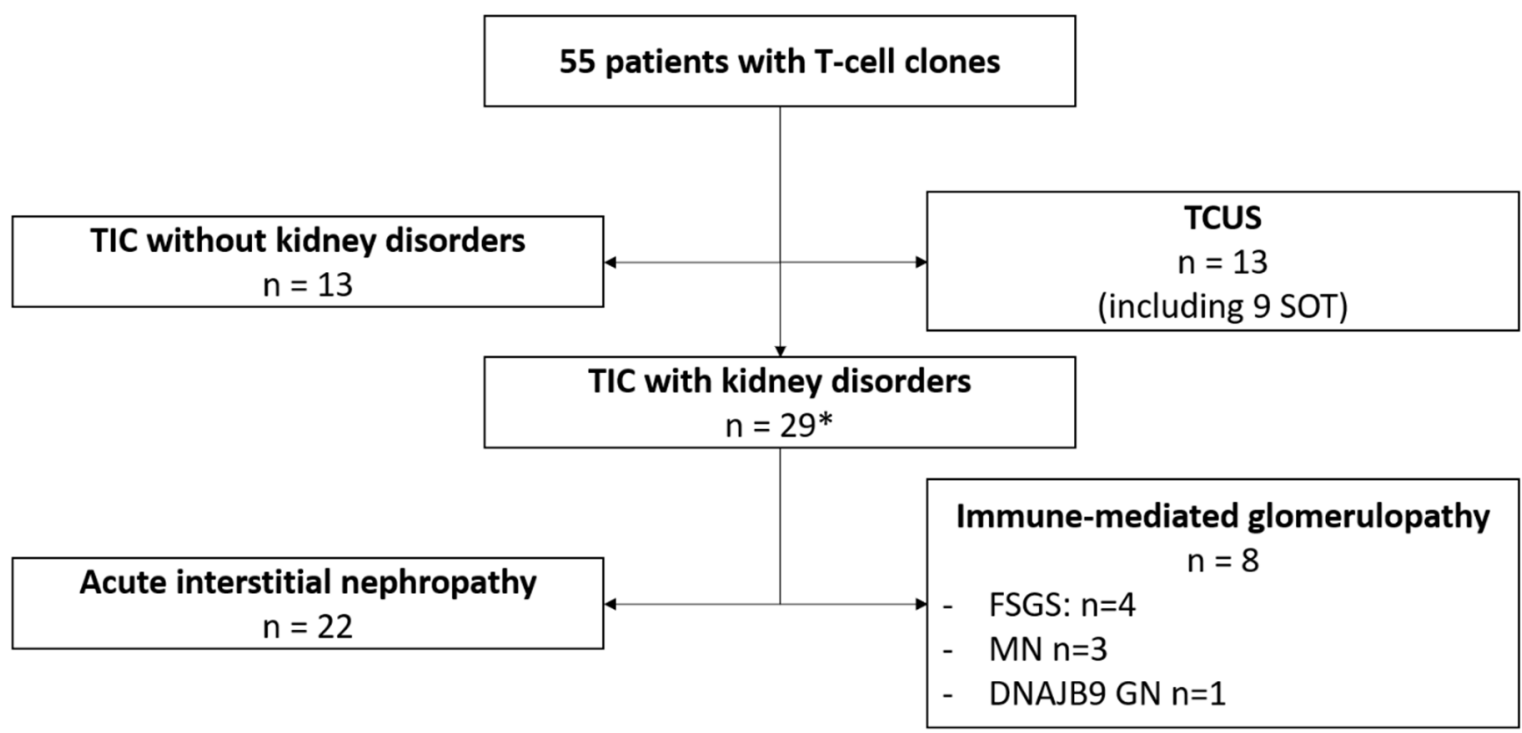

Figure 1. Flow-chart of the study. TIC, T-cell immunoclone; TCUS, T-cell clone of unknown significance; FSGS, focal and segmental glomerulosclerosis; MN, membranous nephropathy; GN, glomerulonephritis. * One patient had both acute interstitial nephropathy and immune-mediated glomerulopathy.

In this study, we addressed the characteristics of 29 out of the 42 TIC patients who developed kidney disease (male gender $n=14,48 \%$; median age at diagnosis 67 years, range 35-84) (Table 1). Twenty patients (49\%) had two or more autoimmune diseases. Six patients had polyclonal hypergammaglobulinemia $(\geq 15 \mathrm{~g} / \mathrm{L})$.

Table 1. Characteristics of the 29 patients with TIC and kidney involvement.

\begin{tabular}{|c|c|c|c|c|c|c|}
\hline $\mathbf{P t}$ & Age & Kidney Disease & Extra-Kidney Disorders & Clone Size & Treatment & eGFR at Last Follow-Up \\
\hline 1 & 70 & Acute interstitial nephropathy & & Tissue & Cst & CR \\
\hline 2 & 62 & Acute interstitial nephropathy & & $23 \%$ & Cst, RTX & $\mathrm{CR}$ \\
\hline 3 & 55 & Acute interstitial nephropathy & Thyroiditis, cholangitis & Tissue & Cst & ESKD \\
\hline 4 & 78 & Acute interstitial nephropathy & $\begin{array}{l}\text { Chronic myelomonocytic } \\
\text { leukemia }\end{array}$ & $16 \%$ & CsA & Stable \\
\hline 5 & 79 & Acute interstitial nephropathy & Hepatitis, chronic neutropenia & $27 \%$ & None & Early death \\
\hline 6 & 52 & Acute interstitial nephropathy & Psoriasis & $20 \%$ & None & Loss of follow-up \\
\hline 7 & 71 & Acute interstitial nephropathy & Interstitial lung disease & $31 \%$ & Cst & Steroid dependency \\
\hline 8 & 76 & Acute interstitial nephropathy & Psoriatic arthritis, pleura infiltration & $27 \%$ & $\begin{array}{l}\text { Leflunomide, } \\
\text { HCQ, MTX, } \\
\text { anti-TNFa, Cst }\end{array}$ & Stable \\
\hline 9 & 49 & Acute interstitial nephropathy & & Tissue & Cst & ESKD \\
\hline 10 & 35 & Acute interstitial nephropathy & Uveitis & $11 \%$ & Cst, CsA & CR \\
\hline 11 & 52 & Acute interstitial nephropathy & IgG4 syndrome & $12 \%$ & Cst, RTX & Steroid dependency \\
\hline 12 & 48 & Acute interstitial nephropathy & & Tissue & Cst & CR \\
\hline 13 & 67 & Acute interstitial nephropathy & & $15 \%$ & Cst & CR \\
\hline 14 & 81 & Acute interstitial nephropathy & & $31 \%$ & Cst & CR \\
\hline 15 & 50 & Acute interstitial nephropathy & & $14 \%$ & Cst, CsA & CR \\
\hline
\end{tabular}


Table 1. Cont.

\begin{tabular}{lllllll}
\hline Pt & Age & Kidney Disease & Extra-Kidney Disorders & Clone Size & Treatment & eGFR at Last Follow-Up \\
\hline 16 & 66 & Acute interstitial nephropathy & IgG4 syndrome & $22 \%$ & Cst, RTX & CR \\
\hline 17 & 69 & Acute interstitial nephropathy & Bullous pemphigoid, cholangitis & $42 \%$ & Cst, CsA & CR \\
\hline 18 & 69 & Acute interstitial nephropathy & $\begin{array}{l}\text { Sjögren syndrome, myasthenia, } \\
\text { interstitial lung disease }\end{array}$ & $20 \%$ & Cst, RTX & Stable \\
\hline 19 & 56 & Acute interstitial nephropathy & Myositis & $14 \%$ & Cst, CsA & ESKD \\
\hline 20 & 46 & Acute interstitial nephropathy & Uveitis, scleritis, thyroiditis & $21 \%$ & Cst & CR \\
\hline 21 & 77 & Acute interstitial nephropathy & $\begin{array}{l}\text { Immune thrombocytopenia, } \\
\text { vitiligo, interstitial lung disease }\end{array}$ & $27 \%$ & Cst, RTX, IgIV, & CR \\
\hline 22 & 84 & Acute interstitial nephropathy & Anemia, thrombocytopenia & $17 \%$ & CSt, CsA & No \\
\hline 23 & 75 & Focal and segmental glomerulosclerosis & & $23 \%$ & CsA & CR \\
\hline & 72 & Focal and segmental glomerulosclerosis & & $21 \%$ & Cst, CsA & CR \\
\hline 24 & 67 & Focal and segmental glomerulosclerosis & & $22 \%$ & Cst, & RTX, Tofacitinib \\
\hline 25 & 43 & PLA2R ${ }^{+}$membranous nephropathy & Immune thrombocytopenia & $7 \%$ & RTX & CR \\
\hline 26 & 71 & PLA2R ${ }^{+}$membranous nephropathy & Polymyalgia rheumatica & $14 \%$ & RTX & CR \\
\hline 27 & 76 & PLA2R ${ }^{+}$membranous nephropathy & Granulomatous hepatitis & $12 \%$ & None & Loss of follow-up \\
\hline 28 & 58 & Minimal change disease & Rheumatoid arthritis & $25 \%$ & Cst, RTX, CsA & CR \\
\hline 29 & 44 & DNAJB9 ${ }^{+}$fibrillary glomerulonephritis & Myositis & $19 \%$ & None & Stable \\
\hline
\end{tabular}

$\mathrm{Pt}$, patient; CR, complete response; Cst, corticosteroids; RTX, rituximab; CsA, ciclosporin-A; HCQ, hydroxychloroquine; MTX, methotrexate; TNFa, tumor necrosis factor-alpha; ESKD, end-stage kidney disease; IgIV, intravenous immunoglobulins; eGFR, estimated glomerular filtration rate.

\subsection{Kidney Presentation}

Among the 29 patients, 22 had acute interstitial nephropathy (i.e., no hypertension, tubular proteinuria, urinary leukocytes, no urinary red blood cells, rapidly progressive renal failure, median estimated glomerular filtration rate at presentation of $22 \mathrm{~mL} / \mathrm{min} / 1.73 \mathrm{~m}^{2}$ (range 0-56)), with non-necrotic granuloma in 6 cases. One patient had both granulomatous interstitial nephropathy and IgG4 ${ }^{+}$PLA2 $^{-}$membranous nephropathy. When assessed, immunostaining showed polymorphic infiltration of inflammatory cells, with predominant $\mathrm{CD}^{+} \mathrm{T}$-cell infiltration without overt CD4/CD8 skewing. Predominant $\mathrm{CD} 3^{+} \mathrm{T}$-cell proliferation did not have the monomorphic pattern usually observed in T-cell lymphoma. In all except two patients, the introduction of a new drug in the last four weeks was ruled out. Of note, the diagnosis of hyper-IgG4 syndrome was ultimately retained in two cases and acute interstitial nephropathy occurred in three patients who previously received an immune checkpoint inhibitor.

Strikingly, a severe inflammatory fibrosis phenotype also involved the liver in three patients, a finding reminiscent of liver, pleura, node, bone marrow, and salivary gland involvement in patients without kidney involvement. Biopsies of extra-kidney involvements showed the same inflammatory pattern.

In eight patients, glomerulopathy was identified, including focal and segmental glomerulosclerosis (FSGS, $n=4)$, membranous nephropathy $(n=3)$, and DNAJB9 ${ }^{+}$fibrillar glomerulonephritis $(n=1)$. In one patient with nephrotic syndrome and FSGS, activated $\mathrm{CD} 8^{+} \mathrm{CD} 56^{+} \mathrm{T}$-cells were shown within the glomerular capillary tuft.

Figure 2 shows representative sections of the kidney biopsies performed in the patients included in our series. 


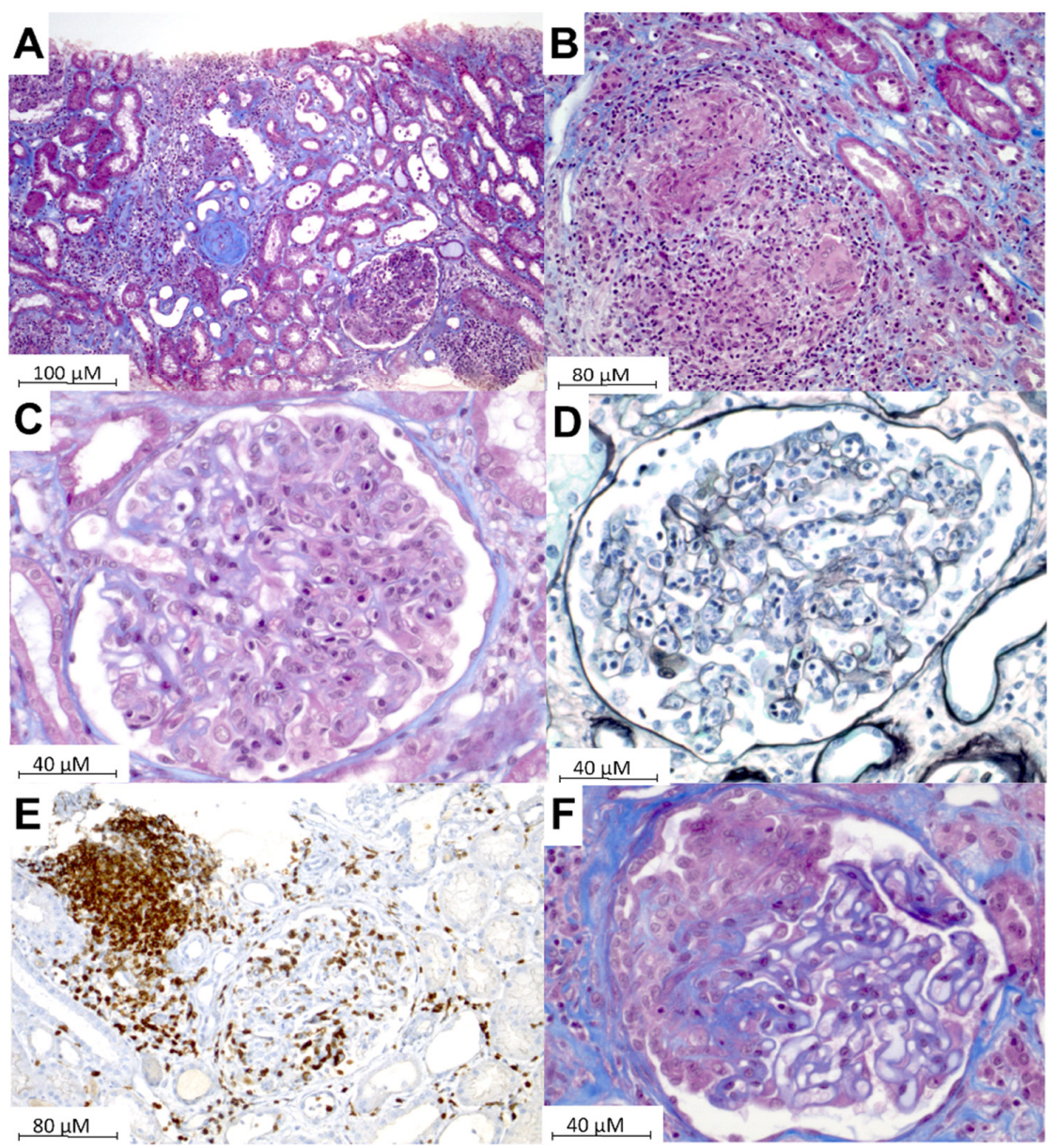

Figure 2. Kidney pathology. (A) Acute interstitial nephropathy with polymorphic infiltration of inflammatory cells and interstitial edema (Masson's trichroma). (B) Interstitial granuloma (Masson's trichroma). (C-E) Glomerulonephritis with CD3+ T-cells' endocapillary proliferation ((C) Masson's trichroma staining, (D) Jones' staining, (E) CD3+ immunostaining in brown). (F) Membranous nephropathy with extra-capillary cell proliferation (Masson's trichroma).

\subsection{Characteristics of the Immunoclones}

Among the 29 patients, all had a normal lymphocyte count. Systematic screening with T-cell immunophenotyping detected the abnormal population (median count of LGL cells at diagnosis $0.32 \times 10^{9} / \mathrm{L}$, range $0.13-3.7$ ). No mutation of STAT3 was identified in the 13 tested patients. The median size of the major clonal population represented $3.7 \%$ of the T-cell population $(0.4 ; 13.1)$, as assessed by next-generation sequencing of the TCRG gene, and represented more than $1 \%$ of the T-cell population in all except two patients.

\subsection{Outcomes}

Frontline therapy included corticosteroids in 23 patients. Three patients did not receive systemic treatment. During follow-up, several lines of an immunosuppressive regimen were used (cyclosporin A $n=8$, rituximab $n=8$, methotrexate $n=1$, tofacitinib $n=2, \mathrm{TNF} \alpha$ inhibitor $n=1$, leflunomide $n=1$ ) but none were associated with complete elimination of the clone. The variety of the immunosuppressive regimen relied on the multiple autoimmune diseases that successively developed in the patients (for instance, rituximab for hyper-IgG4 syndrome or membranous nephropathy). Cyclosporin A was introduced in nine patients because of corticosteroids' refractoriness, dependency, or adverse effects, and led to a dramatic kidney response in four. Cyclosporin was stopped in these four individuals because of the adverse events and/or a lack of efficacy. The JAK inhibitor tofacitinib was 
administered in some patients owing to its activity in refractory large granular lymphocytes leukemia [20].

\subsection{Mechanisms of Inflammatory Fibrosis}

The identification of inflammatory fibrosis, sometimes accompanied by granuloma, prompted us to characterize the activity of the JAK/STAT pathway, a potential therapeutic target in both LGL and granulomatosis [20,21]. Phospho-STAT3 immunostaining was performed on kidney samples from six patients, and in extra-kidney samples from seven additional patients (liver $n=5$, lymph node $n=2$ ), and showed various states of STAT3 activation in lymphocytes in most biopsies (ranging from 1\% to $80 \%$ of lymphocytes, Figure 3 ). In some patients, positive phospho-STAT3 staining was also observed in endothelial or renal tubular cells.

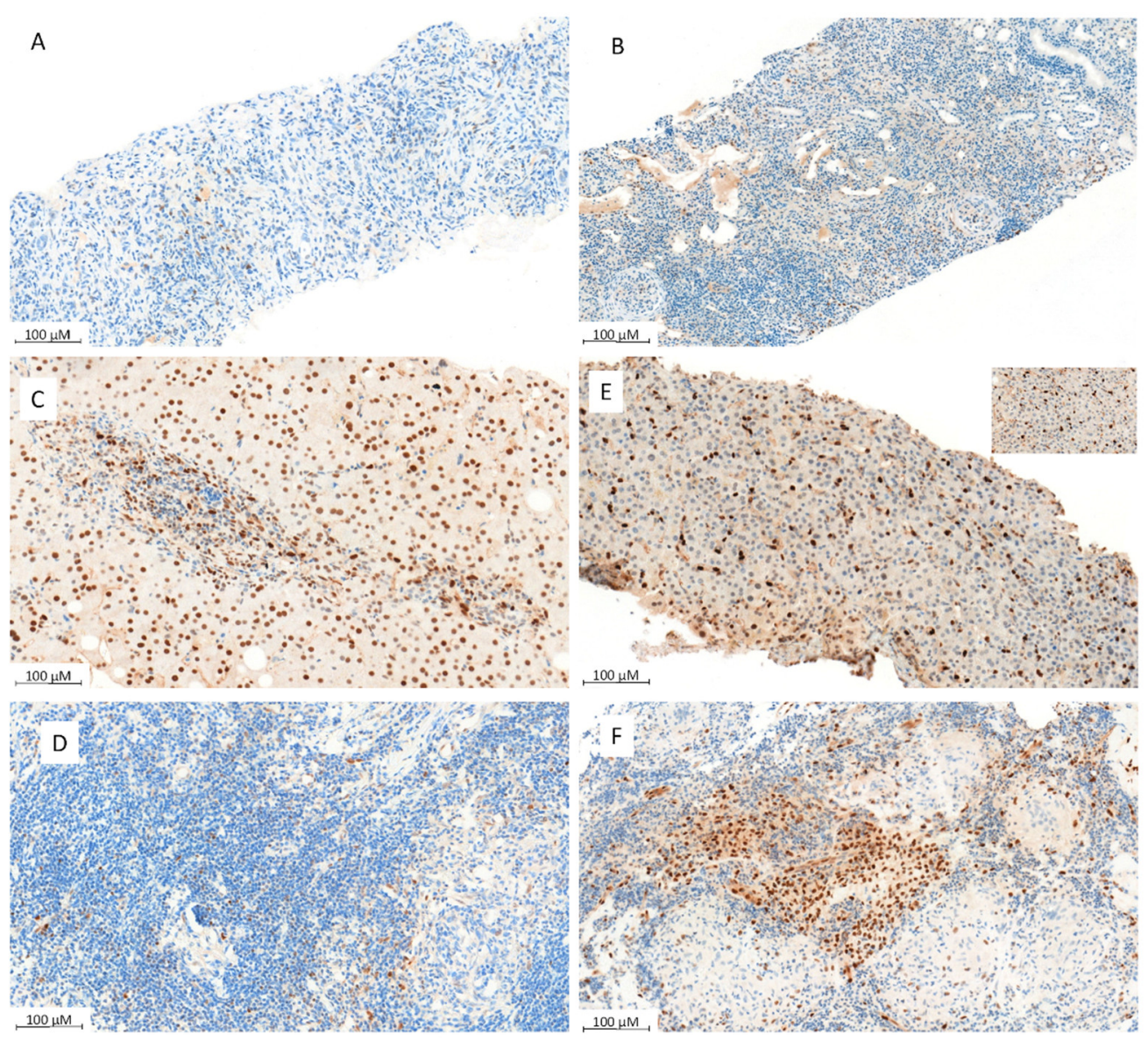

Figure 3. Phospho-STAT3 immunostaining. (A,B) Representative cases of pSTAT3 staining in renal fibrosis showing nuclear expression in some lymphocytes and endothelial cells $(\mathbf{A} \times 150$ and $\mathbf{B} \times 100)$. (C,D) Representative cases of pSTAT3 staining in a liver sample expressed by numerous lymphocytes and hepatocytes $(\mathbf{C} \times 200)$ or highlighting the intra-sinusoidal T-LGL infiltrate $(\mathbf{D} \times 150)$. $(\mathbf{E}, \mathbf{F})$ Representative cases of pSTAT3 staining in inflammatory fibrosis tissue $(\mathbf{E} \times 100)$ and sarcoidosis-like lymph node $(\mathbf{F} \times 150)$ showing nuclear expression mostly in reactive lymphocytes $(\mathbf{D})$ and histiocytes (F) as well as in endothelial cells.

In one patient with severe immune thrombocytopenia refractory to splenectomy, corticosteroids, rituximab, and thrombopoietin agonists, who developed vitiligo and kidney and node granulomatosis while receiving monthly injections of intravenous immunoglobulins, the kidney biopsy showed interstitial nephropathy with intense phospho-STAT3 staining in lymphocytes and tubular cells (Figure 3). Administration of the oral JAK inhibitor tofacitinib was followed by dramatic improvement and led to complete remission 
of thrombocytopenia and vitiligo, but also of the hypermetabolic nodal target (as assessed by a 18F-FDG PET-CT scan), along with kidney function stabilization and immunoglobulins withdrawal.

\section{Discussion}

Despite major advances in the understanding of immune diseases, underlying biological mechanisms remain mostly elusive, precluding individualized and targeted therapy. Combining immunophenotyping, next-generation sequencing of the TCR CDR3 region, and pathology analyses, we showed that LGL proliferation and immunoclones are associated with various immune kidney disorders, including relapsing inflammatory kidney fibrosis with granulomatosis. The link between the LGL proliferation and the antibody-mediated glomerulopathy was more elusive and will require further studies. Additionally, how the detection of a circulating T-cell clone in a kidney transplant recipient may modify its follow-up and immunosuppressive treatment remains elusive. Our study suggests that, in a subset of patients, these clones are of clinical significance and might be targeted to avoid recurrent episodes of acute interstitial nephritis and to improve the long-term prognosis.

Owing to a high clonal proliferation index and a defect of apoptosis, LGL may lead to organomegaly, pancytopenia, and fatigue (LGL leukemia), but in an independent fashion, the production of inflammatory cytokines by LGL cells can promote autoimmunity and BM fibrosis [1]. This was recognized for a long time in patients with LGL leukemia and immune cytopenia or rheumatism. However, the role of small-sized LGL clones remained a matter of debate in patients with other immune disorders [15]. In our series, most patients had no lymphocytosis, and the size of the clonal population was below $0.5 \times 10^{9} / \mathrm{L}$ in most patients, but the LGL population represented more than $1 \%$ of the circulating lymphocytes in almost all TIC patients with kidney involvement. Autoimmunity developed no matter the size of the clonal population. The development of several autoimmune diseases over several years in some patients, especially immune disorders previously associated with LGL [1], also supports the role of small-sized T-cell clones in autoimmunity or organ injury. This paradigm shift was already recognized in monoclonal B-cell lymphocytosis and plasma cell dyscrasia and led to the individualization of monoclonal gammopathy of renal significance and the development of new treatment algorithms [22]. Further studies will now have to refine the frequency of T-cell clones of renal significance and the clinical presentations that should prompt the search for LGL circulating cells.

One of the main findings of our study is the demonstration of LGL cells in several patients referred for interstitial nephropathy. Kidney pathology showed interstitial infiltration of inflammatory cells, especially $\mathrm{CD}^{+} \mathrm{T}$-cells, sometimes accompanied by tubulitis and granuloma. Contrasting with kidney localization of T-cell lymphoma [23], infiltration with polymorphic immune cells suggestive of drug-induced nephropathy should be ruled out first. Sarcoidosis was formerly considered in some patients included in this series. Of note, the angiotensin-converting enzyme was not increased, and the outcome was favorable following cyclosporin A administration in four patients. The recent description of the activation of the JAK/STAT pathway in cutaneous T-cells of patients with refractory sarcoidosis [21] suggests common pathophysiological mechanisms and should prompt the search for LGL cells in atypical, frequently relapsing, or refractory sarcoidosis. It also points out how combined characterization of the circulating immune cell subpopulations and the activation state of tissue lymphocytes may help to tailor the immunosuppressive regimen of patients with complex autoimmune diseases. Hence, our study paves the way for an interventional study testing the efficacy and tolerance of JAK/STAT inhibition in patients with immune kidney disease and T-cell immunoclones [20]. Of note, because circulating T-cell clones may lead to organ injury with overt clonal infiltration (i.e., due to toxic soluble factors' production or to modulation of other T-cell populations prone to induce autoimmunity), assessment of the JAK/STAT pathway activation is probably not the best way to identify patients who will respond to these drugs. 
This study has some limitations, mainly related to its retrospective design. TCR sequencing was not available for all the patients included in the study and the evolution of the clone size could not be followed. Lastly, we could not isolate T-cell clones to address how they promote inflammatory organ fibrosis or which molecular pathways are involved. However, our findings now furnish a tremendous opportunity to better understand the immune-epithelial crosstalk within kidneys and identify new targetable fibrosis pathways.

\section{Conclusions}

We have shown that circulating T-cell clones that are otherwise asymptomatic can promote immune kidney disorders, including a phenotype characterized by severe inflammatory kidney fibrosis and lymphocytic JAK/STAT activation. We suggest performing dedicated flow cytometry of T-cells or searching for T-cell clonality within injured tissues in patients with polyautoimmunity and/or with acute interstitial nephropathy of unknown origin. Targeting the JAK/STAT pathway with the JAK inhibitor tofacitinib in this setting should be tested in a prospective trial.

Author Contributions: S.F. and D.R. designed the study; A.P. and S.F. collected the data; F.V., S.L. and J.-B.R. performed flow cytometry; N.P. performed next-generation sequencing; A.P., J.B., A.H., A.D.B., N.K., L.O., D.C., L.Y., D.R. and S.F. followed the patients; M.C. and C.L. performed pathological and Biomed-2 analyses; S.F. and A.P. wrote the manuscript. All authors have read and agreed to the published version of the manuscript.

Funding: This research received no external funding.

Institutional Review Board Statement: The study was conducted according to the guidelines of the Declaration of Helsinki, and was conducted according to the recommendations of the Institutional Review Board of the University Hospital of Toulouse.

Informed Consent Statement: According to the recommendations of our Institutional Review Board, patients provided oral informed consent to be included in the study.

Data Availability Statement: The data that support the findings of this study are available from S.F., but restrictions apply to the availability of these data, which were used under license for the current study, and so are not publicly available. Data are, however, available from the authors upon reasonable request and with permission from S.F.

Acknowledgments: This study was presented at the annual congress of the Société Francophone de Néphrologie, Dialyse et Transplantation (Lille, 2019).

Conflicts of Interest: No authors had competing interests within the field of this study.

\section{References}

1. Lamy, T.; Moignet, A.; Loughran, T.P. LGL leukemia: From pathogenesis to treatment. Blood 2017, 129, 1082-1094. [CrossRef]

2. Clemente, M.J.; Wlodarski, M.W.; Makishima, H.; Viny, A.D.; Bretschneider, I.; Shaik, M.; Bejanyan, N.; Lichtin, A.E.; Hsi, E.D.; Paquette, R.L.; et al. Clonal drift demonstrates unexpected dynamics of the T-cell repertoire in T-large granular lymphocyte leukemia. Blood 2011, 118, 4383-4393. [CrossRef] [PubMed]

3. Jerez, A.; Clemente, M.J.; Makishima, H.; Rajala, H.; Gómez-Seguí, I.; Olson, T.; McGraw, K.; Przychodzen, B.; Kulasekararaj, A.; Afable, M.; et al. STAT3 mutations indicate the presence of subclinical T-cell clones in a subset of aplastic anemia and myelodysplastic syndrome patients. Blood 2013, 122, 2453-2459. [CrossRef] [PubMed]

4. Kerr, C.M.; Clemente, M.J.; Chomczynski, P.W.; Przychodzen, B.; Nagata, Y.; Adema, V.; Visconte, V.; Lichtin, A.E.; Mustjoki, S.; Radivoyevitch, T.; et al. Subclonal STAT3 mutations solidify clonal dominance. Blood Adv. 2019, 3, 917-921. [CrossRef] [PubMed]

5. Jerez, A.; Clemente, M.J.; Makishima, H.; Koskela, H.; LeBlanc, F.; Ng, K.P.; Olson, T.; Przychodzen, B.; Afable, M.; Gomez-Segui, I.; et al. STAT3 mutations unify the pathogenesis of chronic lymphoproliferative disorders of NK cells and T-cell large granular lymphocyte leukemia. Blood 2012, 120, 3048-3057. [CrossRef] [PubMed]

6. Zhang, M.; Guan, N.; Zhu, P.; Chen, T.; Liu, S.; Hao, C.; Xue, J. Recurrent anti-GBM disease with T-cell large granular lymphocytic leukemia: A case report. Medicine 2019, 98, e16649. [CrossRef] [PubMed]

7. Fu, J.; Lee, L.X.; Zhou, P.; Fogaren, T.; Varga, C.; Comenzo, R.L. A case of T-cell large granular lymphocytic leukemia and renal immunoglobulin heavy chain amyloidosis. Am. J. Case Rep. 2019, 20, 43-47. [CrossRef] 
8. Christopoulos, P.D.; Katsoudas, S.; Androulaki, A.; Nakopoulou, L.; Economopoulos, T.; Vlahakos, D.V. T-cell large granular lymphocyte leukemia presenting as end-stage renal disease: The diagnostic role of flow-cytometric and clonality analysis of the urine sediment. Clin. Nephrol. 2009, 71, 198-202. [CrossRef]

9. Sibal, J.; Valenzuela, R.; Tubbs, R.; Hodge, E.; Nally, J. Successful Renal Transplantation in a Patient with Large Granular Lymphocytic Leukemia with Natural Killer Cell Proliferation. Nephron 1996, 72, 292-297. [CrossRef]

10. Airò, P.; Rossi, G.; Facchetti, F.; Marocolo, D.; Garza, L.; Lanfranchi, A.; Prati, E.; Brugnoni, D.; Malacarne, F.; Cattaneo, R. Monoclonal expansion of large granular lymphocytes with a CD4+ CD8dim+/- phenotype associated with hairy cell leukemia. Haematologica 1995, 80, 146-149.

11. Bassan, R.; Rambaldi, A.; Abbate, M.; Biondi, A.; Allavena, P.; Barbui, T.; Bertani, T. Association of NK-cell lymphoproliferative disease and nephrotic syndrome. Am. J. Clin. Pathol. 1990, 94, 334-338. [CrossRef] [PubMed]

12. Hotta, O.; Miyazawa, S.; Taguma, Y.; Onodera, S.; Kitamura, H.; Yusa, N.; Nakamura, K. Mesangiolysis associated with severe glomerular endocapillary proliferation of CD57 large granular lymphocytes. Clin. Nephrol. 1995, 43, 297-302. [PubMed]

13. Ribes, D.; Casemayou, A.; El Hachem, H.; Laurent, C.; Guilbeau-Frugier, C.; Vergez, F.; Tavitian, S.; Schanstra, J.P.; Chauveau, D.; Bascands, J.L.; et al. Asymptomatic circulating T-cell clone cause renal polymorphic inflammatory fibrosis. Clin. Exp. Nephrol. 2017, 21, 781-786. [CrossRef]

14. Mailloux, A.W.; Zhang, L.; Moscinski, L.; Bennett, J.M.; Yang, L.; Yoder, S.J.; Bloom, G.; Wei, C.; Wei, S.; Sokol, L.; et al. Fibrosis and Subsequent Cytopenias Are Associated with Basic Fibroblast Growth Factor-Deficient Pluripotent Mesenchymal Stromal Cells in Large Granular Lymphocyte Leukemia. J. Immunol. 2013, 191, 3578-3593. [CrossRef] [PubMed]

15. Singleton, T.P.; Yin, B.; Teferra, A.; Mao, J.Z. Spectrum of Clonal Large Granular Lymphocytes (LGLs) of $\alpha \beta$ T Cells: T-Cell Clones of Undetermined Significance, T-Cell LGL Leukemias, and T-Cell Immunoclones. Am. J. Clin. Pathol. 2015, 144, 137-144. [CrossRef] [PubMed]

16. Dhodapkar, M.V.; Li, C.Y.; Lust, J.A.; Tefferi, A.; Phyliky, R.L. Clinical spectrum of clonal proliferations of T-large granular lymphocytes: A T-cell clonopathy of undetermined significance? Blood 1994, 84, 1620-1627. [CrossRef] [PubMed]

17. Bigouret, V.; Hoffmann, T.; Arlettaz, L.; Villard, J.; Colonna, M.; Ticheli, A.; Gratwohl, A.; Samii, K.; Chapuis, B.; Rufer, N.; et al Monoclonal T-cell expansions in asymptomatic individuals and in patients with large granular leukemia consist of cytotoxic effector T cells expressing the activating CD94:NKG2C/E and NKD2D killer cell receptors. Blood 2003, 101, 3198-3204. [CrossRef]

18. Van Dongen, J.J.M.; Langerak, A.W.; Brüggemann, M.; Evans, P.A.S.; Hummel, M.; Lavender, F.L.; Delabesse, E.; Davi, F.; Schuuring, E.; García-Sanz, R.; et al. Design and standardization of PCR primers and protocols for detection of clonal immunoglobulin and T-cell receptor gene recombinations in suspect lymphoproliferations: Report of the BIOMED-2 Concerted Action BMH4-CT98-3936. Leukemia 2003, 17, 2257-2317. [CrossRef] [PubMed]

19. Duez, M.; Giraud, M.; Herbert, R.; Rocher, T.; Salson, M.; Thonier, F. Vidjil: A web platform for analysis of high-Throughput repertoire sequencing. PLoS ONE 2016, 11, e0166126. [CrossRef]

20. Bilori, B.; Thota, S.; Clemente, M.J.; Patel, B.; Jerez, A.; Afable, M., II; Maciejewski, J.P. Tofacitinib as a novel salvage therapy for refractory T-cell large granular lymphocytic leukemia. Leukemia 2015, 29, 2427-2429. [CrossRef]

21. Damsky, W.; Thakral, D.; Emeagwali, N.; Galan, A.; King, B. Tofacitinib Treatment and Molecular Analysis of Cutaneous Sarcoidosis. N. Engl. J. Med. 2018, 379, 2540-2546. [CrossRef] [PubMed]

22. Fermand, J.P.; Bridoux, F.; Dispenzieri, A.; Jaccard, A.; Kyle, R.A.; Leung, N.; Merlini, G. Monoclonal gammopathy of clinical significance: A novel concept with therapeutic implications. Blood 2018, 132, 1478-1485. [CrossRef] [PubMed]

23. Kohn, M.; Karras, A.; Zaidan, M.; Bénière, C.; de Fréminville, J.B.; Laribi, K.; Perrin, M.C.; Malphettes, M.; le Calloch, R.; Anglaret, B.; et al. Lymphomas with kidney involvement: The French multicenter retrospective LyKID study. Leuk. Lymphoma 2020, 61, 887-895. [CrossRef] [PubMed] 